\title{
Stochastic Simulation of Inhomogeneous Metocean Fields. Part I: Annual Variability
}

\author{
Alexander V. Boukhanovsky ${ }^{1}$, Harald E. Krogstad ${ }^{2}$, \\ Leonid J. Lopatoukhin ${ }^{3}$, and Valentin A. Rozhkov ${ }^{3}$ \\ ${ }^{1}$ Institute for High Performance Computing and Information Systems, \\ St. Petersburg, Russia \\ avb@fn.csa.ru, http://www.csa.ru \\ ${ }^{2}$ Dept. Mathematical Sciences, NTNU, Trondheim, Norway \\ harald.krogstad@math.ntnu.no \\ ${ }^{3}$ Oceanology Dept., State University, St. Petersburg, Russia \\ leonid@LL1587.spb.edu
}

\begin{abstract}
The paper discusses stochastic models of scalar and vector metocean fields based on time varying Empirical Orthogonal Functions in space, and autoregressive time series models for the coefficients in the expansions. The models are fitted to an extensive data set from the Barents Sea and verified by studying field extreme value properties.
\end{abstract}

\section{Introduction}

Annual variations of spatial and temporal properties are characteristic features of most metocean fields, and today several approaches for statistical analyses and modeling of such fields have been developed. For example, in the papers of Dragan et al. [4] and Lopatoukhin $\mathrm{el} \mathrm{al}$. [13] the time series of air and water temperature, river run-off, wind speed, wave heights and sea level variations have been treated as Periodically Correlated Stochastic Processes (PCSP). For spatial fields, periodical representations based on Empirical Orthogonal Functions (EOFs) have also been proposed by Kim\&Wu [10] and Kim\&North, [11].

In the present paper we shall study the annual variations of metocean events as inhomogeneous random fields by constructing probabilistic description of their spatial and annual variabilities. The aims of the current research have been to

- develop computationally efficient procedures for stochastic simulation of ensembles of annual variations of metocean fields;

- apply the above mentioned procedures to numerical investigations of the field extremes. 


\section{The Data Set}

All computations carried out by the stochastic models have been tested against an extensive data set from the Barents Sea region. The input data set consists of fields of atmospheric sea level pressure (SLP), wind speed (WS) and air temperature (AT) from the NCEP/NCAR reanalysis data [9]. The input wave fields are numerically obtained fields calculated by Wave Watch model [15]. A summary of sampling and duration of the data sets are presented in Table 1.

Table 1. Sampling and duration characteristics of the data set. A Gaussian grid with latitude variable step is used for air temperature and wind speed.

\begin{tabular}{|c|c|c|c|c|c|}
\hline & Field & Time step & $\begin{array}{c}\text { Spatial } \\
\text { Sampling }\end{array}$ & Duration & Source \\
\hline 1 & Sea level pressure & $6 \mathrm{~h}$ & $2.5^{\circ} \times 2.5^{\circ}$ & 1948-1999 & \multirow{3}{*}{$\begin{array}{l}\text { NCEP/ } \\
\text { NCAR }\end{array}$} \\
\hline 2 & Air temperature & $24 \mathrm{~h}$ & $\Delta \lambda=1.875^{\circ}$ & 1958-1997 & \\
\hline 3 & Wind speed & $6 \mathrm{~h}$ & $\overline{\Delta \varphi} \approx 1.9^{\circ}$ & 1948-1999 & \\
\hline 4 & Wind waves & $6 \mathrm{~h}$ & $0.5^{\circ} \times 1.5^{\circ}$ & 1971-1997 & $\begin{array}{c}\text { Wave } \\
\text { Watch III }\end{array}$ \\
\hline
\end{tabular}

\section{Analysis of Inhomogeneous Metocean Fields}

The second order inhomogeneity of a metocean field $\zeta(\mathbf{r}, t)$ is defined in terms of the mean value $m_{\zeta}(\mathbf{r}, t)=\mathrm{E} \zeta(\mathbf{r}, t)$, and the covariance function

$$
K_{\zeta}\left(\mathbf{r}_{1}, \mathbf{r}_{2}, t, \tau\right)=\mathrm{E}\left\{\zeta^{0}\left(\mathbf{r}_{1}, t\right) \zeta^{0}\left(\mathbf{r}_{2}, t+\tau\right)\right\}
$$

where ${ }^{0}$ denotes the centering operation, $X^{0}=X-\mathrm{E}(X)$. Alternatively, the covariance function may be defined as $K_{\zeta}\left(\mathbf{r}_{1}, \boldsymbol{\rho}, t, \tau\right)$, where $\mathbf{r}=(x, y)$ are the geographical coordinates and $\boldsymbol{\rho}=\mathbf{r}_{2}-\mathbf{r}_{1}$ the spatial lag. The large scale inhomogeneity is due to variations in the spatial distribution of atmospheric or ocean forcing.

A preliminary analysis of the data shows that during winter, a low mean SLP, $m_{S L P}(\mathbf{r}, t)$, in the western part of the Barents sea is prevailing with an increasing pressure towards north, south and east. In summer, the situation is the opposite with the highest pressure in the center of the sea, and dropping in all directions away from the center. However, during all the year, the sea level pressure is below standard $(1013.2 \mathrm{hPa})$. The standard deviation $\sigma_{S L P}=K_{S L P}(\mathbf{r}, \mathbf{r}, t, 0)^{1 / 2}$ of the SLP variations is about $8 \mathrm{hPa}$ in winter, and only $2.5-3.5 \mathrm{hPa}$ in summer.

The standard deviation of the air temperature in the center of sea in winter is increasing from $2^{\circ}$ to $6^{\circ}$ toward north, whereas the variability in summer is fairly constant around $2.5-3.5^{\circ}$. 
We may conclude, based on the variations of $m_{\zeta}(\mathbf{r}, t)$ and $\sigma_{\zeta}(\mathbf{r}, t)$, that these metocean fields are inhomogeneous in space as well as non-stationary in time. In fact, seasonal changes in the spatial patterns is a typical feature of the spatio-temporal variability of metocean fields. Since the physical interpretation of the full covariance function is harder than for the functions $m_{\zeta}(\mathbf{r}, t), \sigma_{\zeta}(\mathbf{r}, t)$, this has lead us to consider EOFs, which are calculated by means of the integral equation

$$
\int_{\Omega} K_{\zeta}\left(\mathbf{r}_{1}, \mathbf{r}_{2}\right)_{t} \varphi_{k t}\left(\mathbf{r}_{2}\right) d \mathbf{r}_{2}=\lambda_{k t} \varphi_{k t}\left(\mathbf{r}_{1}\right)
$$

where $\lambda_{k t}$ are eigenvalues and $K_{\zeta}\left(\mathbf{r}_{1}, \mathbf{r}_{2}\right)_{t}=K_{\zeta}\left(\mathbf{r}_{1}, \mathbf{r}_{2}, t, 0\right)$ [12]. The first and second EOFs for the SLP are shown in Fig. 1.

The covariance function for vector fields $\mathbf{V}(\mathbf{r}, t)$ like wind, currents, and temperature gradients, is defined

$$
\mathbf{K}_{\mathbf{V}}(\mathbf{r}, \boldsymbol{\rho}, t, \tau)=\mathrm{E}\left\{\mathbf{V}^{0}(\mathbf{r}, t) \otimes \mathbf{V}^{0}(\boldsymbol{\rho}, \tau)\right\},
$$

where $\otimes$ is the tensor (or outer) product of $\mathbf{V}^{0}=\mathbf{V}-\mathbf{m}_{\mathbf{V}}[1]$. Note that $\mathbf{K}_{\mathbf{V}}(\mathbf{r}, \boldsymbol{\rho})_{t}$ is a tensor,

$$
\left(\begin{array}{ll}
K_{u} & K_{u v} \\
K_{v u} & K_{v}
\end{array}\right)
$$

where $K_{u}, K_{u v}, K_{v}$ are auto- and cross-covariance functions of the components $\left(\mathbf{V}_{x}, \mathbf{V}_{y}\right)$ of $\mathbf{V}$ in geographical coordinates. Similarly to Eqn. (2), vector EOFs (VEOFs) may be computed by a tensor integral equation

$$
\int_{\Omega} \mathbf{K}_{\mathbf{V}}\left(\mathbf{r}_{1}, \mathbf{r}_{2}\right)_{t} \boldsymbol{\Psi}_{k t}\left(\mathbf{r}_{2}\right) d \mathbf{r}_{2}=\lambda_{k t} \boldsymbol{\Psi}_{k t}\left(\mathbf{r}_{1}\right)
$$

where $\boldsymbol{\Psi}_{k}=\varphi_{k} \mathbf{i}+\psi_{k} \mathbf{j}$ and $(\mathbf{i}, \mathbf{j})$ are the unit vectors in geographical coordinates [3]. The first VEOFs of wind speed for January and July are shown in Fig. 1. We observe that not only the EOFs of sea level pressure, but also the VEOFs for the wind change quite significantly from winter to summer.

The EOFs and VEOFs are both orthogonal with respect to the inner product defined by the covariance function, and may therefore be used as basis functions for an orthogonal expansion of the fields. Thus, the field $\zeta(\mathbf{r}, t)$ may be expanded as

$$
\zeta(\mathbf{r}, t)-m(\mathbf{r}, t)=\sum_{k} a_{k}(t) \varphi_{k t}(\mathbf{r}, t)
$$

The basis functions $\varphi_{k t}(\mathbf{r}, t)$ are periodic in time, and may thus be considered as generalizations of the component representation of a field discussed in Dragan et al. [4],

$$
\zeta(t)=\sum_{k} \xi_{k}(t) \exp \left(-i \Lambda_{k} t\right)
$$


where $\xi_{k}(t)$ are the stationary random functions, $\Lambda_{k}=2 \pi k / T$ are frequencies, and $T$ the fundamental period of variation (1 year).

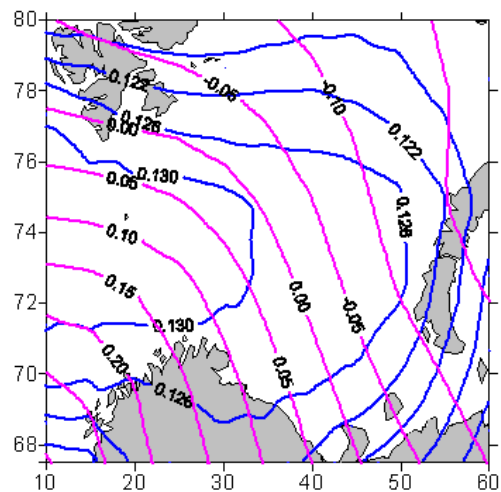

(a)

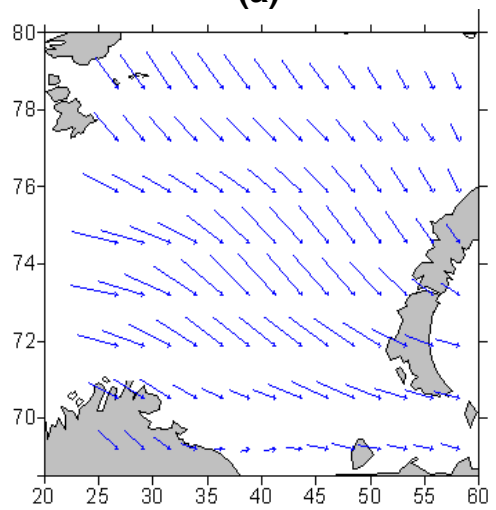

(c)

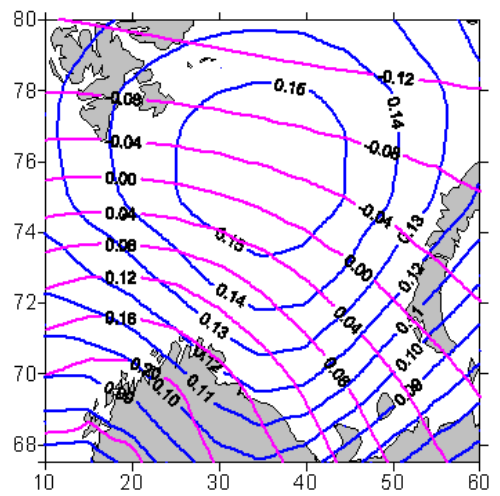

(b)

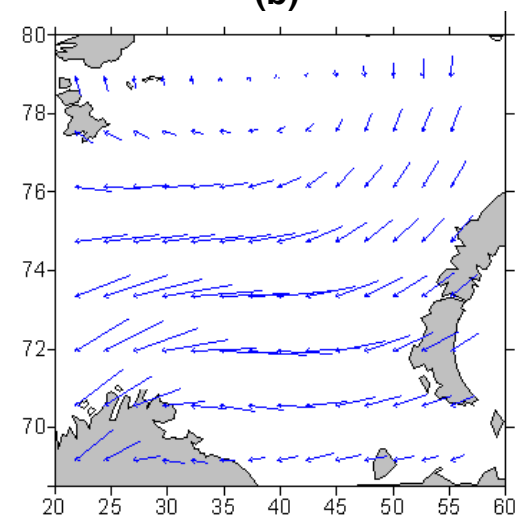

(d)

Fig. 1. Periodical EOFs of metocean fields: SLP: $(a, b)$ and WS $(c, d)$ for different months: January $(a, c)$ and July $(c, d)$. (Black - first EOF of SLP, Gray - second one)

If we estimate EOFs $\varphi_{k t}(\mathbf{r})$ on the basis of the averaged spatial covariance function over the year, we obtain an all months basis $\varphi_{\mathrm{k}}(\mathbf{r})$. The annual variations will then only be manifested in the coefficients $a_{k}(t)$ of Eqn. (5), as illustrated for the SLP auto- and cross correlation functions $K_{a_{k}}(\tau)$ in Fig. 2. It is seen that the autocorrelation in $a_{k}(t)$ is much smaller when we use the seasonal EOFs. Moreover, in that case, the cross correlation between the different time functions $a_{k}(t)$ become negligible. An estimate of the convergence of the series in Eqn. (5) for SLP, air temperature, wave heights and wind speed, calculated by the mean of the ratio $\lambda_{i} / \sum_{k} \lambda_{k}$ is shown in Table 2. 
Table 2. Estimates of the speed of convergence (in \%) of the orthogonal expansions in Eqn. (5) of various metocean fields by EOFs. Representative months (I,IV,VII and XI) for the periodic basis functions.

\begin{tabular}{|c|c|c|c|c|c|}
\hline \multirow{2}{*}{$\mathrm{k}$} & \multicolumn{4}{|c|}{ Periodic basis $\varphi_{k t}(\vec{r})$} & \multirow{2}{*}{$\begin{array}{l}\text { Time- } \\
\text { independent } \\
\text { basis } \varphi_{k}(\vec{r}) \\
\end{array}$} \\
\hline & I & IV & VII & XI & \\
\hline \multicolumn{6}{|c|}{ Sea level pressure } \\
\hline 1 & 84.0 & 81.8 & 67.7 & 77.5 & 82.0 \\
\hline 2 & 8.3 & 8.8 & 19.6 & 12.0 & 7.7 \\
\hline 3 & 5.3 & 6.3 & 8.0 & 6.9 & 7.3 \\
\hline \multicolumn{6}{|c|}{ Air temperature } \\
\hline 1 & 57.5 & 60.9 & 43.8 & 64.7 & 89.8 \\
\hline 2 & 17.6 & 17.1 & 21.8 & 14.6 & 3.3 \\
\hline 3 & 8.8 & 6.3 & 10.7 & 7.0 & 3.2 \\
\hline \multicolumn{6}{|c|}{ Wind wave heights } \\
\hline 1 & 75.4 & 70.0 & 65.6 & 75.7 & 70.6 \\
\hline 2 & 10.0 & 13.3 & 13.2 & 9.6 & 22.3 \\
\hline 3 & 2.5 & 7.5 & 8.3 & 10.9 & 5.3 \\
\hline \multicolumn{6}{|c|}{ Wind speed (at $10 \mathrm{~m}$ level) } \\
\hline 1 & 39.1 & 42.6 & 53.5 & 46.8 & 28.2 \\
\hline 2 & 19.7 & 25.3 & 17.0 & 28.9 & 26.0 \\
\hline 3 & 17.1 & 12.1 & 11.0 & 9.0 & 20.8 \\
\hline
\end{tabular}

It seen from Table 2, that the first term in Eqn. (5) holds 40-85\% of the total variance of the field values. A sum of the first three terms in (5) holds about $85-95 \%$ of the variance. Let us also note that different orthogonal polynomial, e.g., Chebyshev, Legendre, spherical harmonics etc. may be used as basis functions in Eqn. (5) [2]. However, the convergence of the EOFs expansion is the best in terms of the second-order metric defined by the covariance function.

\section{The Stochastic Simulation Procedure}

Taking into account Table 2, the relation in Eqn. (5) may be written as

$$
\zeta(\mathbf{r}, t)=m(\mathbf{r}, t)+\sum_{k} a_{k}(t) \varphi_{k t}(\mathbf{r}, t)+\varepsilon(\mathbf{r}, t)
$$

where $N$ is a representative number of terms, and $\varepsilon(\mathbf{r}, t)$ is residual white noise (both in space and time). The noise variance is typically not more than $20 \%$ of $\sigma_{\zeta}^{2}$. Bearing in mind the type of autocovariance functions $K_{a_{k}}(\tau)$ shown in Fig. 3, and ignoring the 
cross correlations defined by $K_{a_{k} a_{j}}(\tau)$, the stochastic processes $a_{k}(t)$ may be presented in the form of uncorrelated scalar autoregressive models [7]:

$$
a_{k}(t)=\sum_{j=1}^{p} \Phi_{j}^{(k)} a_{k}(t-j)+\delta^{(k)}(t)
$$

Here the $\Phi_{j}^{(k)}$ coefficients are calculated from the covariance function $K_{a_{k}}(\tau)$, and $\delta^{(k)}(t)$ is a constant variance Gaussian white noise, only dependent on $k$. The relations (7) and (8) constitute a stochastic model of the inhomogeneous (by $\mathbf{r}$ ) and periodically correlated (by $t$ ) random field $\zeta(\mathbf{r}, t)$.
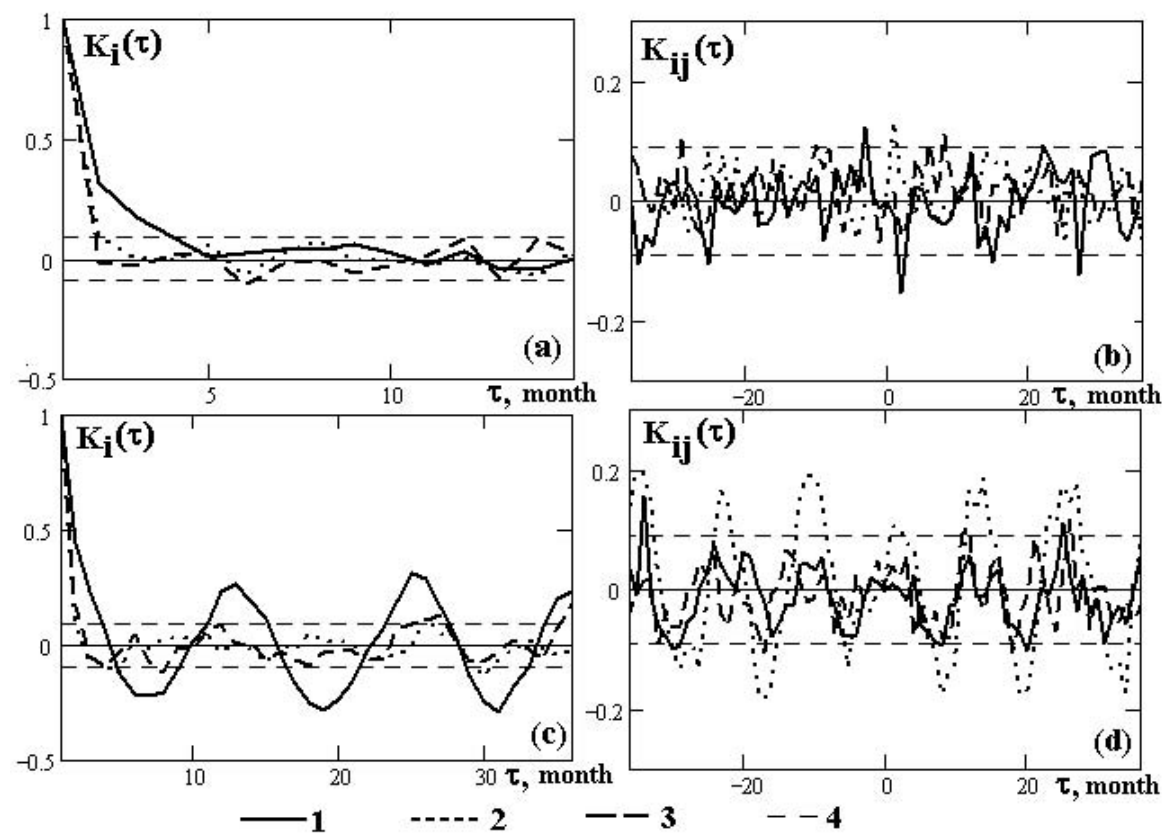

Fig. 2. Auto- $(a, b)$ and cross $(b, d)$ covariance functions (AKF, CKF) for the expansion coefficients in Eqn. (7) of SLP field by periodic basis $(a, b)$ and averaged by months basis $(c, d)$. For the AKF, $1-3$ denotes the serial number of the coefficient. For the CKF, 1 corresponds to $(i=2$, $j=1), 2$ to $(i=3, j=1)$, and 3 to $(i=3, j=2)$. Moreover, 4 are the $90 \%$ limits confidence interval for the estimates.

The functions $a_{k}(t)$ and $\varepsilon(\mathbf{r}, t)$ in Eqn. (7) enable us to regard it both as a regression and a factor model [8]. The terminology of multidimensional statistical analysis is not always suitable for the analysis of random processes and fields. E.g., the model in Eqn. (7) is known as a regression, but not as a factor model, although $a_{k}(t)$ are random functions, and, moreover, $\left\{\Phi_{i}^{(k)}\right\}$ are calculated by means of $K_{a_{k}}(\tau)$. The functions 
$\varphi_{k t}(\mathbf{r})$, calculated by Eqns. (1) and (3), are known as EOFs, and not as the factor loadings.

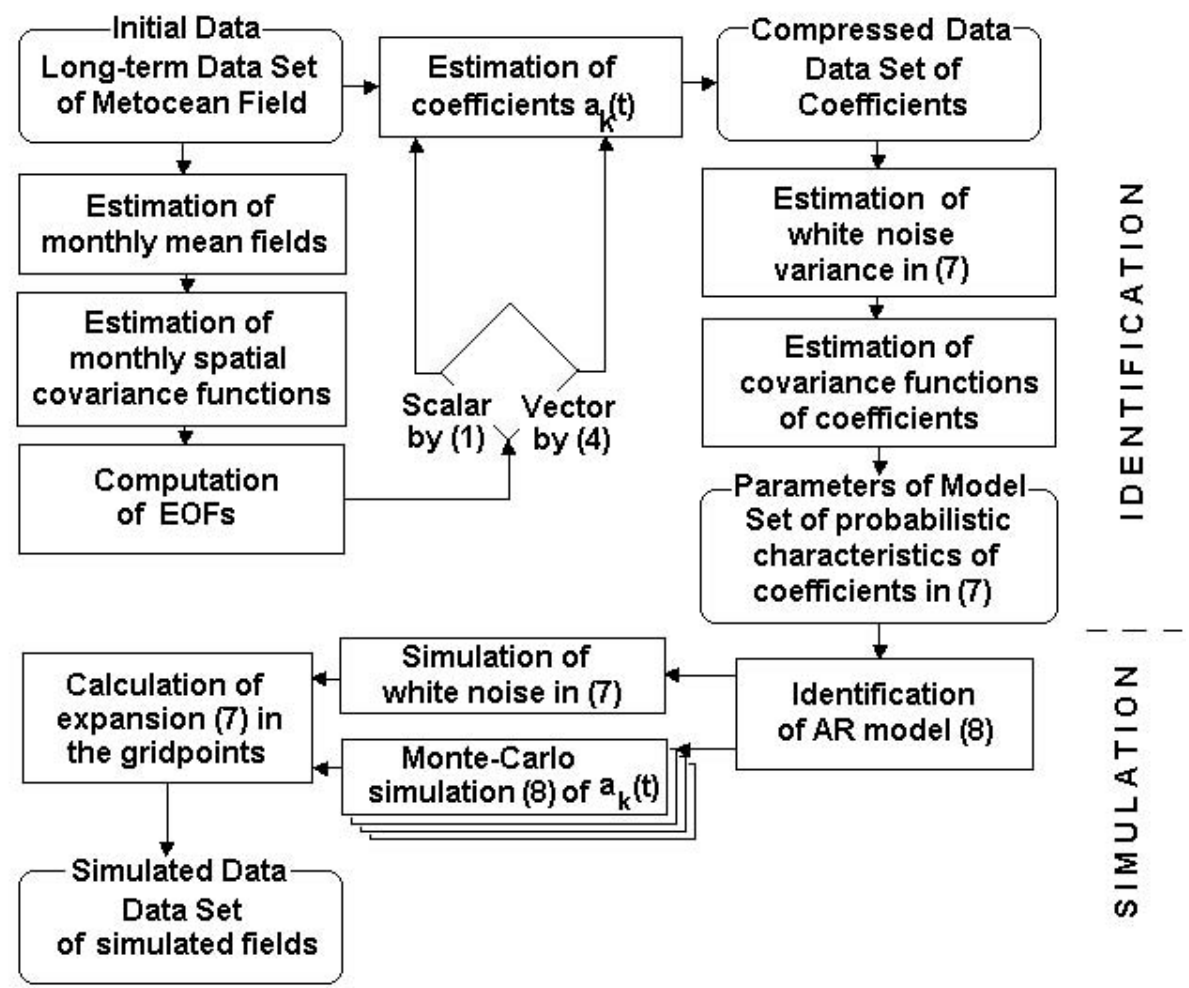

Fig. 3. Stochastic simulation of annual variation of inhomogeneous metocean fields: general algorithm.

The general algorithm of the stochastic model identification and simulation is shown in Fig. 3. The model identification starts by estimating $\{m(\mathbf{r}, t)\}$ and $\left\{\varphi_{k t}(\mathbf{r})\right\}$ for a discrete set of grid points on a monthly basis. By expanding the fields into EOF series by means of $\left\{\varphi_{k t}(\mathbf{r})\right\}$, the remaining time variations is reduced to the time series of scalar coefficients $\left\{a_{k}(t)\right\}$. The coefficient time series, which now are stationary, are then in turn fitted to suitable autoregressive models by invoking the covariance functions $K_{a_{k}}(\tau)$. The estimation of autoregressive coefficients $\Phi_{j}^{(k)}$ in Eqn. (8) for each time series $a_{k}(t)$ is discussed in numerous places, e.g. in [7]. Finally, the variance of the white noise field $\varepsilon(\mathbf{r}, t)$ is calculated.

When using the model for simulations, an ensemble of independent realizations of $a_{k}(t)$ for the time span $[0, T]$ of the computations, is first produced by Eqn. (8). Then, the realizations of the full spatiotemporal metocean fields are obtained by 
means substituting of the simulated series of $a_{k}(t)$ into Eqn. (7) and adding the white noise term.

The result of the simulation procedure is the set of the simulated metocean fields $\zeta(\mathbf{r}, t)$ or $\mathbf{V}(\mathbf{r}, t)$ in a discrete set of grid points $\mathbf{r}_{i j}=\left(x_{i}, y_{j}\right)$ and at discrete times, $\left\{t_{n}\right\}$.

\section{Verification and Extreme Value Analysis}

The developed simulation procedure may be verified and tested by considering the problem of spatial extremes. In practice, due to inhomogeneity of the fields, there arise questions of joint extremes in two or more spatial points. A Gaussian kernel estimate [14] of the joint distribution of mean monthly minima of SLP in the SW and E parts of Barents Sea is presented in Table 3 (left). This estimation was obtained from the initial data set given in Table 1. It is seen that although the two parts of the sea are more than $1000 \mathrm{~km}$ from each other, the correlation is rather high (about 0.7).

Since extreme value properties were not considered as a part of the model identification, the extreme values predictions by the model provide independent model verification. In the right part of the Table 3, a similar Gaussian kernel estimate of joint SLP minima in the same points have been obtained from simulations using Eqns. 7 and 8. It is observed that the maxima of the joint probability are located on the main diagonal, and this reflects the overall correlation of the sea level pressure field. The differences between probabilities are insignificant. Even the largest difference between $p_{i, j}^{(1)}=31.5 \%$ and $p_{i j}^{(2)}=17.1 \%$ is inside the $90 \%$ confidence interval.

Table 3. Kernel estimates of joint distributions of sea level pressure minima in the points $\left(\varphi=70^{\circ} \mathrm{N}, \lambda=15^{\circ} \mathrm{E}\right)$ and $\left(\varphi=76^{\circ} \mathrm{N}, \lambda=45^{\circ} \mathrm{E}\right)$.

\begin{tabular}{|c|c|c|c|c|c|c|c|c|c|c|}
\hline \multirow{4}{*}{$\begin{array}{c}\varphi=70^{\circ} \\
\mathrm{N}, \\
\lambda=15^{\circ} \mathrm{E} \\
(\mathrm{hPa})\end{array}$} & \multicolumn{5}{|c|}{ Sample data } & \multicolumn{5}{|c|}{ Probability model (Eqns. 7,8) } \\
\hline & \multicolumn{10}{|c|}{$\varphi=76^{\circ} \mathrm{N}, \lambda=45^{\circ} \mathrm{E}(\mathrm{hPa})$} \\
\hline & 5 & 0 & -5 & -10 & -15 & 5 & 0 & -5 & -10 & -15 \\
\hline & 0 & -5 & -10 & -15 & -20 & 0 & -5 & -10 & -15 & -20 \\
\hline $2-2$ & 10.9 & 7.5 & 0.5 & - & - & 15.9 & 10.1 & 0.1 & - & - \\
\hline$-2-6$ & 6.2 & 17.1 & 4.1 & 0.1 & - & 6.7 & 31.5 & 1.8 & - & - \\
\hline$-6-10$ & 1.8 & 15.2 & 10.1 & 0.3 & - & 0.6 & 14.3 & 8.4 & 1.1 & - \\
\hline$-10-14$ & 0.2 & 4.6 & 9.6 & 1.1 & 0.3 & - & 0.7 & 3.6 & 1.7 & 0.2 \\
\hline$-14-18$ & - & 0.9 & 5.2 & 2.3 & 1.4 & - & 0.0 & 0.9 & 1.7 & 0.7 \\
\hline
\end{tabular}

Annual extremes, calculated from monthly mean values of water temperature and salinity, sea level, wave heights, may in different years be observed in different months. Thus, the distribution for the annual extreme, $F_{Y}(x)$, will then a mixture of distributions for the monthly mean $F_{i}(x)$ [13] with the weights $\rho_{i}-$ the probabilities of appearance of $X_{\min }$ or $X_{\max }$ in the month $i$.

We may now pose two questions: 
Does the distribution in Eqn. (9) extend from a single point extreme to a field extreme?

Does the model in Eqns. (7,8) reproduce both the distribution (9) and its field modification?

Table 4 answers these questions in the affirmative. We observe a quite good agreement between sample and simulated extremes over the entire field. E.g., from the data only in $7.5 \%$ of the mean monthly temperatures in the Barents Sea is below $-39^{\circ}$. By the model simulations, this value has changed to $5.5 \%$, which, however, is well inside $90 \%$ confidence limits. A similar agreement is observed for the probability $\rho_{i}$ of monthly extremes. E.g., the annual lowest air temperature over the Barents Sea occurs in January $40 \%$ of the time, whereas this occurs $45 \%$ of the time in the simulations, again well inside the $90 \%$ confidence limits.

Table 4. Probability characteristics of annually varying metocean fields

\begin{tabular}{|c|c|c|c|c|c|c|c|c|c|c|}
\hline \multicolumn{11}{|c|}{ Minimum of sea level pressure (hPa-1000) } \\
\hline \multirow[t]{2}{*}{ Data } & \multicolumn{7}{|c|}{ Probability by months $\rho_{i}(\%)$} & \multicolumn{3}{|c|}{$\begin{array}{c}\text { Integral } \\
\text { probability }(\%)\end{array}$} \\
\hline & IX & $\mathrm{X}$ & $\mathrm{XI}$ & XII & $\mathrm{I}$ & II & III & $<0$ & $<-5$ & $<-15$ \\
\hline Sample & 2.5 & 10 & 20 & 20 & 20 & 15 & 12.5 & 95 & 75 & 1.5 \\
\hline $\begin{array}{l}\text { 90\% Confi- } \\
\text { dence interval }\end{array}$ & $\begin{array}{c}0 \\
7.4\end{array}$ & $\begin{array}{l}0.5 \\
9.5\end{array}$ & $\begin{array}{c}7.3 \\
36.2\end{array}$ & $\begin{array}{c}7.3 \\
36.2\end{array}$ & $\begin{array}{c}7.3 \\
36.2\end{array}$ & $\begin{array}{c}3.7 \\
26.3\end{array}$ & $\begin{array}{c}2.0 \\
23.0\end{array}$ & $\begin{array}{c}88.1 \\
100\end{array}$ & $\begin{array}{c}61 . \\
3 \\
88 . \\
7\end{array}$ & $\begin{array}{c}0 \\
5.3\end{array}$ \\
\hline Model $(7,8)$ & - & 7.5 & 15 & 30 & 20 & 15 & 12.5 & 95 & 63 & $<1$ \\
\hline \multicolumn{11}{|c|}{ Minima of air temperature $\left({ }^{0} \tilde{\mathrm{N}}\right)$} \\
\hline \multirow{2}{*}{ Data } & \multicolumn{7}{|c|}{ Probability by months $\rho_{i}(\%)$} & \multicolumn{3}{|c|}{$\begin{array}{l}\text { Integral probability } \\
(\%)\end{array}$} \\
\hline & IX & $\mathrm{X}$ & XI & XII & I & II & III & $<-30$ & $<-33$ & $\begin{array}{l}<- \\
39\end{array}$ \\
\hline Sample & - & - & 2.5 & 15 & 40 & 27.5 & 15 & 90 & 47.5 & 7.5 \\
\hline $\begin{array}{l}90 \% \text { Confi- } \\
\text { dence interval }\end{array}$ & - & - & $\begin{array}{c}0 \\
7.4\end{array}$ & $\begin{array}{c}3.7 \\
26.3\end{array}$ & $\begin{array}{l}24.5 \\
55.5\end{array}$ & $\begin{array}{l}13.4 \\
41.6\end{array}$ & $\begin{array}{c}3.7 \\
26.3\end{array}$ & $\begin{array}{l}80.5 \\
99.5\end{array}$ & $\begin{array}{l}31.7 \\
63.3\end{array}$ & $\begin{array}{r}0 \\
15 . \\
8 \\
\end{array}$ \\
\hline Model $(7,8)$ & - & - & 5 & 12.5 & 45 & 27.5 & 10 & 95 & 70 & 5.5 \\
\hline
\end{tabular}

\section{Conclusions}

This paper has shown that computational multivariate statistics of spatio-temporal fields may be used for describing the metocean regime in seas and oceans.

By applying field expansions in terms of periodical EOFs and VEOFs, it has been possible to develop stochastic models which are field generalizations of PCSPs.

The models may be applied to simulate ensembles of time varying metocean fields like SLP, WS, AT, and sea waves.

Investigation of spatial and temporal features of annual extremes of measured and simulated metocean fields may be used for independent model verification. Further applications will be discussed in a separate paper in these proceedings. 
Acknowledgment. This research has been partly founded by INTAS 99-0666 Project: "Estimation of extreme metocean events".

\section{References}

1. Belyshev A.P., Klevantsov Yu.P., Rozhkov V.A. Probabilistic analysis of sea currents. Leningrad, Gymet P.H., (1983) 264 p. (in Russian)

2. Blais J.A.R. Estimation and spectral analysis. Univ. of Calgary Press, (1988) 132 p.

3. Boukhanovsky A.V., Degtyarev A.B., Rozhkov V.A. Peculiarities of computer simulation and statistical representation of time-spatial metocean fields. LNCS \#2073, SpringerVerlag, (2001) 463-472

4. Dragan Ya. P., Rozhkov V.A., Yavorsky I.N. Methods of probabilistic analysis of rhythms of oceanological processes. Leningrad, Gymet. P.H., (1987) 320 p. (in Russian)

5. Dubrovin B.A., Novikov S.P., Fomenko A.T. Modern geometry: methods and applications. Moscow, "Nauka", (1979) 760 p. (in Russian)

6. Isihara A. Statistical physics. Acad. Press, New York-London, (1971) 472 p.

7. Jenkins G.M., Watts D.G. Spectral analysis and its application. Holden-Day, SanFrancisco (1969)

8. Johnson R.A., Wichern D.W. Applied multivariate statistical analysis. Prentice-Hall Internalional, Inc., London (1992) 642 p.

9. Kalnay E., M. Kanamitsu, R. Kistler, W. Collins, D. Deaven, L. Gandin, M. Iredell, S. Saha, G. White, J. Woollen, Y. Zhu, A. Leetmaa, R. Reynolds, M. Chelliah, W. Ebisuzaki, W.Higgins, J. Janowiak, K. C. Mo, C. Ropelewski, J. Wang, R. Jenne, D. Joseph. The NCEP/NCAR 40-Year Reanalysis Project. Bulletin of the American Meteorological Society, ${ }^{13}$, March (1996)

10. Kim, K.-Y., and G. R. North, EOFs of harmonizable cyclostationary processes, J. Atmos. Sci., 54 (1997) 2416-2427

11. Kim, K.-Y., and Q. Wu, A comparison study of EOF techniques: Analysis of nonstationary data with periodic statistics, J. Clim. 12 (1999) 185-199

12. Loeve M. Fonctions aleatories de second odre. C.R. Acad. Sci. 220, (1945)

13. Lopatoukhin L.J., Rozhkov V.A., Ryabinin V.E., Swail V.R., Boukhanovsky A.V., Degtyarev A.B. Estimation of extreme wave heights. JCOMM Technical Report, WMO/TD \#1041 (2000)

14. Silverman B.W. Density estimation for statistics and data analysis. London, Chapman \& Hall, (1986)

15. Tolman H. User manual and system documentation of WAVE WATCH-III. NOAA technical note. (1999) $124 \mathrm{p}$. 